\title{
Application Of Extended Kalman Filter For A Free Falling Body Towards Earth
}

\author{
Leela Kumari. B \\ Department of Electronics and communication \\ Engineering \\ G.V.P. College of Engineering, \\ Visakhapatnam, India \\ Email:leela8821@yahoo.com
}

\author{
Sai Krishna. R \\ Department of Electronics and \\ communication Engineering \\ G.V.P. College of Engineering \\ Visakhapatnam, India \\ Email: rskrishna89@gmail.com
}

\author{
Chandan .V.Y.V \\ Department of Electronics and \\ G.V.P. College of Engineering \\ Visakhapatnam, India \\ Email:chandanvyv964@gmail.com
}

\author{
Padma Raju. K \\ Department of Electronics and communication \\ Engineering \\ J. N.T.U.KAKINADA \\ Kakinada, India \\ Email:padmaraju_K@yahoo.com
}

\begin{abstract}
State estimation theory is one of the best mathematical approaches to analyze variants in the states of the system or process. The state of the system is defined by a set of variables that provide a complete representation of the internal condition at any given instant of time. Filtering of Random processes is referred to as Estimation, and is a well-defined statistical technique. There are two types of state estimation processes, Linear and Nonlinear. Linear estimation of a system can easily be analyzed by using Kalman Filter (KF) and is used to compute the target state parameters with a priori information under noisy environment. But the traditional KF is optimal only when the model is linear and its performance is well defined under the assumptions that the system model and noise statistics are well known. Most of the state estimation problems are nonlinear, thereby limiting the practical applications of the KF. The modified KF, aka EKF, Unscented Kalman filter and Particle filter are best known for nonlinear estimates. Extended Kalman filter (EKF) is the nonlinear version of the Kalman filter which linearizes about the current mean and covariance. The estimation can be linearised around the current estimate using the partial derivatives to compute estimates even in the face of nonlinear relationships.. The EKF has been considered the standard in the theory of nonlinear state estimation. This paper deals with how to estimate a nonlinear model with Extended Kalman filter (EKF). The approach in this paper is to analyze Extended Kalman filter where EKF provides better probability of state estimation for a free falling body towards earth.
\end{abstract}

Keywords- Kalman filter; Extended Kalman filter; free fall body; apriori information.

\section{INTRODUCTION}

Filtering and estimation are two of the most important tools of engineering. Whenever the state of a system needs to be estimated from noisy sensor information, state estimator is employed to produce the best estimate of the true system state. When the system dynamics and observation models are linear, the minimum mean squared error (MMSE) estimate can be computed using the Kalman filter.

Control of any process modeling, obtained from a priori knowledge of certain observable parameters is standard practice for Engineers. For many of the applications simple models with linear approximations around a design point suffice the requirement. Since all the natural phenomena are non-linear, it is very important to study the nonlinear models and their control for the following reasons:

1) Some systems have a linear approximation that is non-controllable near interesting working points. Linearization is ineffective even locally for such cases.

2) Even if the linearized model is controllable one may wish to extend the operational domain beyond the validity domain into nonlinear region for better prediction.

3) Some control problems are external to the process and cannot be answered by a linearly approached model.

The success of the linear model in identification or in control has its cause in the good understanding of it. A better mastery of invariants of nonlinear models for some transformations is a prerequisite to a true theory of nonlinear identification and control. And all nonlinear systems are supposed to have a state space of finite dimension. State Estimation techniques are handled by filtering technique models for performance.

A common approach to overcome this problem is to linearize the system before using the Kalman filter, resulting in an extended Kalman filter. This linearization does however pose some problems, e.g. it can result in nonrealistic estimates $[1,2]$ over a period of time. The development of better estimator algorithms for nonlinear Systems has therefore attracted a great deal of interest in the scientific community, 
because the improvements will undoubtedly have great impact in a wide range of engineering fields. The EKF has been considered the standard in the theory of nonlinear state estimation. This paper deals with how to estimate a nonlinear model with Extended Kalman filter (EKF). The approach in this paper is to analyze Extended Kalman filter where EKF provides better probability of state estimation for a free falling body towards earth.

\section{LINEAR AND NONLINEAR MODELS}

Kalman Filter (KF), Extended KF (EKF), Unscented KF (UKF) and Particle filter (PF) are models popularly used for state estimation.

The traditional Kalman Filter is optimal only when the model is linear. . The practical application of the KF is limited because most of the state estimation problems like tracking of the target are nonlinear. If the system is linear, the state estimation parameters like the mean and covariance can be exactly updated with the KF.

The EKF works on the principal that a linearized transformation is approximately equal to the true nonlinear transformation.

In this paper, EKF for State Estimation have been considered for their relative performance levels and to give an idea as to their applications with sample State Estimation case study.

\section{A. State space models}

A state space model is a mathematical model of a process, where state $\mathbf{x}$ of a process is represented by a numerical vector. State-space model actually consists of two sub models: the process model, which describes how the state propagates in time based on external influences, such as input and noise; and the measurement model, which describe how measurements $\mathbf{z}$ are taken from the process, typically simulating noisy and/or inaccurate measurements.

\section{B. Linear State Space Model}

A linear state-space model assumes the functions $F$ and $H$ are linear, in both state and input. The functions can then be expressed by using the matrices, $B$ and $H$, reducing state propagation calculations to linear algebra. Overall this results in the following state-space model:

$$
\begin{aligned}
& \mathbf{X}_{k}=F_{k} \mathbf{X}_{k-1}+B_{k} \mathbf{u}_{k-1}+\mathbf{W} k-1 \\
& \mathbf{Z}_{k}=H_{k} \mathbf{X} k+\mathbf{V} k
\end{aligned}
$$

Where

$\mathbf{u}$ is process input

$\mathbf{w}$ is state vector

$\mathbf{v}$ is measurement noise vector

$k$ is the discrete time

The above expressions (1) and (2) govern state propagation and measurements respectively.

Linear model is easier both to calculate and analyze. This enables modelers to investigate properties such as controllability, observability and frequency response [11].
Linear state models are either based on inherently linear processes, or simply linearized versions of a nonlinear process by means of a first order Taylor approximation.

\section{Nonlinear State Space Model}

The most general form of state-space models is the Nonlinear model. This model does typically consist of two functions, $f$ and $h$ :

$$
\begin{aligned}
& \mathbf{x} k=f(\mathbf{x} k-1, \mathbf{u} k-1, \mathbf{w} k-1) \\
& \mathbf{z} k=h(\mathbf{x} k, \mathbf{v} k)
\end{aligned}
$$

\section{EXTENDED KALMAN FILTER}

\section{A. Back ground-State estimation}

State estimation concerns the problem of estimating the probability density function (pdf) for the state of a process which is not directly observable. This involves both predicting the next state (based on the current state) and applying corrections (based on measurement model).

Estimator: Estimator is a tool that predicts the future behavior of a model from the available information.

The Estimator uses knowledge about the evaluation of the variable, the probabilistic characterization of the various random factors and the prior information.

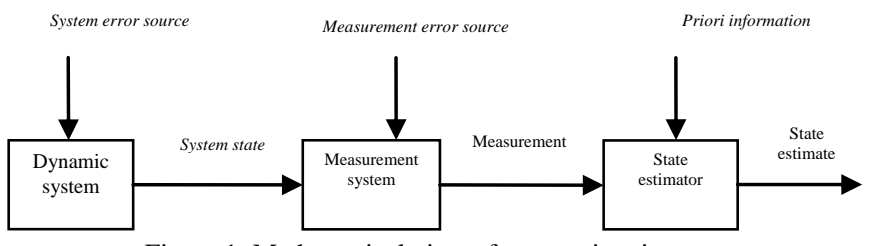

Figure 1: Mathematical view of state estimation

Different estimators:

- Recursive Bayesian Estimation

- Kalman Filter (KF)

. Extended KF (EKF)

- Unscented KF (UKF) and

- Particle filter (PF)

\section{B. Recursive Bayesian Estimation(RBE) State Space}

The most general form of state estimation is known as Recursive Bayesian Estimation [12]. This is the optimal way of predicting a state pdf for any process, given a system and a measurement model. RBE works by simulating the process, while at the same time adjusting it to account for new measurements $\mathrm{z}$, taken from the real process. The calculations are performed recursively in a two-step procedure. First, the next state is predicted by extrapolating the current state onto next time step, using state propagation belief $\mathrm{p}\left(\mathrm{x}_{\mathrm{k}} \mid \mathrm{x}_{(\mathrm{k}-1)}\right)$ obtained from function $\mathrm{f}$. Secondly, this prediction is corrected using measurement likelihood $\mathrm{p}\left(\mathrm{z}_{\mathrm{k}} \mid \mathrm{x}_{\mathrm{k}}\right)$ obtained from function $\mathrm{h}$, taking new measurements into account. Unfortunately, this method does not scale very well in practice, mainly due to the large state space for multidimensional state vectors. Calculating the prior probability of each point in this state space involves a 
multidimensional integral, which quickly becomes intractable as the state space grows. Computers are also limited to calculation of the pdf in discrete point in state space, requiring a discretization of the state space. This technique is therefore mainly considered as a theoretic foundation for state estimation in general. Bayesian estimation by means of computers is only possible if either the state space can be discretized, or if certain limitations apply for the model.

\section{Kalman filter}

The problem of state estimation can be made tractable if we put certain constrains on the process model, by requiring both ' $\mathrm{f}$ ' and ' $\mathrm{h}$ ' to be linear functions, and the Gaussian and white noise terms ' $\mathrm{w}$ ' and ' $\mathrm{v}$ ' to be uncorrelated, with zero mean. Put in mathematical notation, we then have the following constraints (5) and (6).

As the model is linear and input is Gaussian, we know that the state and output will also be Gaussian [13]. The state and output pdf will therefore always be normally distributed, where mean and covariance are sufficient statistics. This implies that it is not necessary to calculate a full state pdf anymore, a mean vector ${ }^{\wedge} \mathrm{X}$ and covariance matrix $\mathrm{P}$ for the state will suffice.

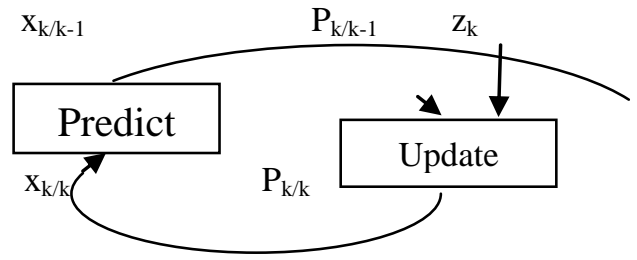

Figure 2: Kalman filter loop

The recursive Bayesian estimation technique is then reduced to the Kalman filter, where $f$ and $h$ is replaced by the matrices F, B and H. The Kalman filter is, just as the Bayesian estimator, decomposed into two steps: predict and update.

The Kalman filter is quite easy to calculate, due to the fact that it is mostly linear, except for a matrix inversion. It can also be proved that the Kalman filter is an optimal estimator of process state, given a quadratic error metric [14, 15].

Most processes in real life are not linear, and therefore need to be linearised before they can be estimated by means of a Kalman filter. So the practical applications of the KF are limited and so modified KF, aka EKF is generally used.

Different from KF, EKF deals with nonlinear process model and nonlinear observation model. In the extended Kalman filter, the state transition and observation models need not be linear functions of the state, but may be differentiable functions $[4,5,6,7,8]$. The nonlinear process model (from time $k$ - 1 to time $k$ ) is described as

$$
\begin{aligned}
& \mathrm{X}_{\mathrm{k}}=\mathrm{f}\left(\mathrm{x}_{\mathrm{k}^{-1}}, \mathrm{u}_{\mathrm{k}^{-1}}\right)+\mathrm{w}_{\mathrm{k}^{-1}} \\
& \mathrm{Z}_{\mathrm{k}}=\mathrm{h}\left(\mathrm{x}_{\mathrm{k}}\right)+\mathrm{v}_{\mathrm{k}}
\end{aligned}
$$

where $\mathrm{x}_{\mathrm{k}-1}, \mathrm{x}_{\mathrm{k}}$ are the system state (vector) at time $\mathrm{k}-1 ; \mathrm{k}, \mathrm{f}$ is the system transition function, $\mathrm{u}_{\mathrm{k}}$ is the control, $\mathrm{w}_{\mathrm{k}}$ is the zero-mean Gaussian process noise $\mathrm{w}_{\mathrm{k}} \sim \mathrm{N}(0 ; \mathrm{Q}), \mathrm{h}$ is the observation function and $\mathrm{v}_{\mathrm{k}+1}$ is the zero-mean Gaussian observation noise $\mathrm{v}_{\mathrm{k}+1} \sim \mathrm{N}(0 ; \mathrm{R})$.
The function $f$ can be used to compute the predicted state from the previous estimate and similarly the function $h$ can be used to compute the predicted measurement from the predicted state. However, $f$ and $h$ cannot be applied to the covariance directly. Instead a matrix of partial derivatives (the Jacobian) is computed.

At each time step the Jacobian is evaluated with current predicted states. These matrices can be used in the Kalman filter equations. This process essentially linearizes the nonlinear function around the current estimate.

\section{Predict and update equations}

Predicted state

$$
\hat{x}_{k ! k-1}^{-}=f\left(\hat{x}_{k-1 ! k-1}^{-}, u_{k-1}\right)
$$

Predicted estimate covariance

$$
P_{k ! k-1}=F_{k-1} P_{k-1 ! k-1} F_{k-1}^{T}+Q_{k-1}
$$

Updating state

Innovation(or residual) covariance

$$
\begin{aligned}
& y_{k}^{\tilde{z}}=z_{k}-h\left(\hat{x}_{k ! k-1}\right) \\
& S_{k}=H_{k} P_{k ! k-1} H_{k}^{T}+R_{k}
\end{aligned}
$$

Optimal Kalman gain

$$
K_{k}=P_{k ! k-1} H_{k}^{T} S_{k}^{-1}
$$

Updated state estimate

$$
\left.\hat{x}_{k ! k}=\hat{x}_{k ! k-1}+K_{k} y_{k}^{\sim}\right)
$$

Updated estimate covariance

$$
P_{k ! k}=\left(1-K_{k} H_{k}\right) P_{k ! k-1}
$$

Where the state transition and observation matrices are defined to be the following Jacobians

$$
\begin{gathered}
F_{k-1}=\frac{\partial f}{\partial x} \mid x_{k-1 \mid k-1}^{\wedge}, u_{k-1} \\
H_{k}=\frac{\partial h}{\partial x} \mid x_{k \mid k-1}^{\wedge}
\end{gathered}
$$

E. Continuous-time extended Kalman filter Model

$$
\begin{aligned}
& \mathrm{x}(\mathrm{t})=\mathrm{f}(\mathrm{x}(\mathrm{t}), \mathrm{u}(\mathrm{t}))+\mathrm{w}(\mathrm{t}), \\
& \mathrm{w}(\mathrm{t}) \sim \mathrm{N}(0, \mathrm{Q}(\mathrm{t})) \\
& \mathrm{z}(\mathrm{t})=\mathrm{h}(\mathrm{x}(\mathrm{t}))+\mathrm{v}(\mathrm{t}), \\
& \mathrm{v}(\mathrm{t}) \sim \mathrm{N}(0, \mathrm{R}(\mathrm{t}))
\end{aligned}
$$


Initialize

$$
\hat{x}\left(t_{0}\right)=E\left[x\left(t_{0}\right)\right], P\left(t_{0}\right)=\operatorname{Var}\left[x\left(t_{0}\right)\right]
$$

Predict-Update

$$
\begin{gathered}
\hat{x}\left(t_{0}\right)=f\left(\hat{x}\left(t_{0}\right), u(t)+K(t)\left(z(t)-h\left(\hat{x}\left(t_{0}\right)\right)\right)\right. \\
P(t)=F(t) P(t)+P(t) F(t)^{T}-K(t) H(t) P(t)+Q(t) \\
F(t)=\frac{\partial f}{\partial x} \mid x^{\wedge}(t), u(t) \\
K(t)=P(t) H(t)^{T} R(t)^{-1} \\
H(t)=\frac{\partial h}{\partial x} \mid x^{\wedge}(t)
\end{gathered}
$$

Unlike discrete-time extended Kalman filter, the prediction and update steps are coupled in continuous-time extended Kalman filter $[9,10]$.

\section{F. Continuous- discrete extended Kalman}

Most physical systems are represented as continuous-time models while discrete-time measurements are frequently taken for state estimation via a digital processor. Therefore, the system model and measurement model are given by

$$
\begin{aligned}
& \mathrm{x}(\mathrm{t})=\mathrm{f}(\mathrm{x}(\mathrm{t}), \mathrm{u}(\mathrm{t}))+\mathrm{w}(\mathrm{t}), \\
& \mathrm{w}(\mathrm{t}) \sim \mathrm{N}(0, \mathrm{Q}(\mathrm{t})) \\
& \mathrm{z}(\mathrm{t})=\mathrm{h}\left(\mathrm{x}_{\mathrm{k}}\right)+\mathrm{v}_{\mathrm{k}}, \\
& \mathrm{v}_{\mathrm{k}} \sim \mathrm{N}\left(0, \mathrm{R}_{\mathrm{k}}\right)
\end{aligned}
$$

where, $x_{k}=x\left(t_{k}\right)$

\section{Initialize}

$\hat{x}_{0 \mid 0}=E\left[x\left(t_{0}\right)\right], P_{0 \mid 0}=\operatorname{Var}\left[x\left(t_{0}\right)\right]$

Predict

$$
\begin{aligned}
& \left\{\begin{array} { l } 
{ \dot { \hat { \mathbf { x } } } ( t ) = f ( \hat { \mathbf { x } } ( t ) , \mathbf { u } ( t ) ) } \\
{ \dot { \mathbf { P } } ( t ) = \mathbf { F } ( t ) \mathbf { P } ( t ) + \mathbf { P } ( t ) \mathbf { F } ( t ) ^ { \top } + \mathbf { Q } ( t ) }
\end{array} \text { , with } \left\{\begin{array}{l}
\hat{\mathbf{x}}\left(t_{k-1}\right)=\hat{\mathbf{x}}_{k-1 \mid k-1} \\
\mathbf{P}\left(t_{k-1}\right)=\mathbf{P}_{k-1 \mid k-1}
\end{array}\right.\right. \\
\Rightarrow & \left\{\begin{array}{l}
\hat{\mathbf{x}}_{k \mid k-1}=\hat{\mathbf{x}}\left(t_{k}\right) \\
\mathbf{P}_{k \mid k-1}=\mathbf{P}\left(t_{k}\right)
\end{array}\right.
\end{aligned}
$$

where,

$$
F(t)=\frac{\partial f}{\partial x} \mid x^{\wedge}(t), u(t)
$$

Update

$$
\begin{aligned}
& K_{k}=P_{k \mid k-1} H_{k}{ }^{T}\left(H_{k} P_{k \mid k-1} H_{k}{ }^{T}+R_{k}\right)^{-1} \\
& \hat{x}_{k ! k}=\hat{x}_{k ! k-1}+K_{k}\left(z-h\left(\hat{x}_{k ! k-1}\right)\right) \\
& P_{k \mid k}=\left(I-K_{k} H_{k}\right) P_{k \mid k-1}
\end{aligned}
$$

where,

$$
H_{k}=\frac{\partial h}{\partial x} \mid \hat{x}_{k \mid k-1}
$$

The update equations are identical to those of discrete-time extended Kalman filter.

\section{MOdELling EXAMPLE FOR A FREE FALLING Body TOWARDS EARTH}

Consider the problem of estimating various states of a free falling body towards earth. Range measurements are corrupted by additive Gaussian noise. The state estimation cannot be accurately explained by KF since nonlinearities are exhibited by forces that act on the body, and the measuring device is located at an altitude $\mathrm{h}$ and the horizontal range between the measuring device and the body is $\mathrm{M}$.

The trajectory parameters are the altitude above the earth's surface (h), velocity (v) and ballistic coefficient (K).

\section{A. Examples of objects not in free fall}

- Flying in an aircraft: there is also an additional force of lift.

- Standing on the ground: the gravitational acceleration is counteracted by the normal force from the ground.

- Descending to the Earth using a parachute, which balances the force of gravity with an aerodynamic drag force (and with some parachutes, an additional lift force).

An initially-stationary object which is allowed to fall freely as shown in fig.3, under gravity drops a distance which is proportional to the square of the elapsed time. This image, spanning half a second, was captured with a stroboscopic flash at 20 flashes per second. During the first 1/20th of a second the ball drops one unit of distance (here, a unit is about $12 \mathrm{~mm}$ ); by 2/20ths it has dropped at total of 4 units; by 3/20ths, 9 units and so on.

Under normal earth-bound conditions, when objects move owing to a constant gravitational force a set of dynamical equations describe the resultant trajectories. For example, Newton's law of universal gravitation simplifies to $F=m g$, where $\mathrm{m}$ is the mass of the body. This assumption is reasonable for objects falling towards earth over the relatively short vertical distances of our everyday experience, but is very much untrue over larger distances, such as spacecraft trajectories. Please note that in this article any resistance from air (drag) is neglected [3]. 


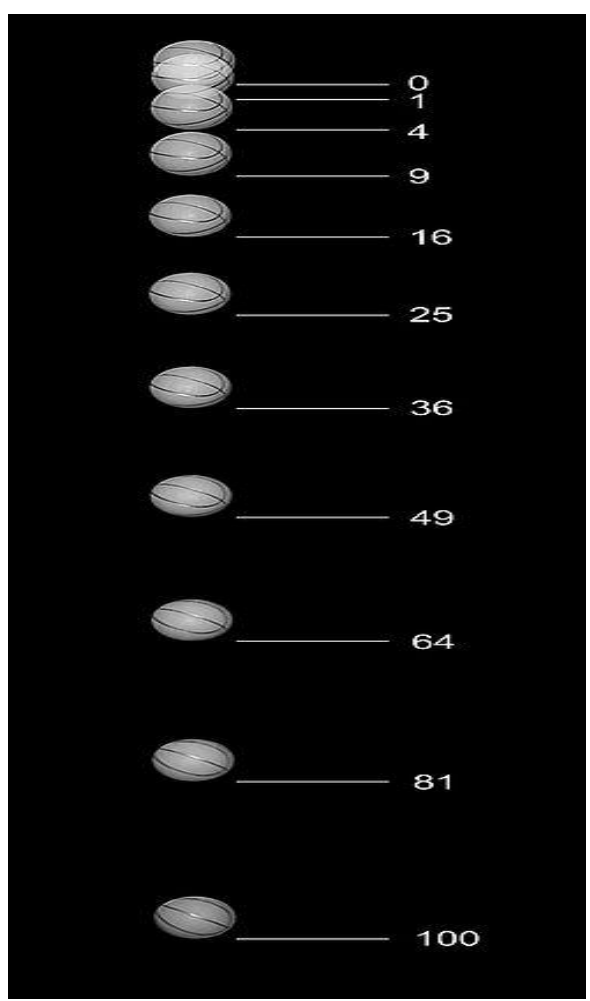

Fig3: Free fall body

\section{B. Development of the filter model}

The state space model of Particle filter is given by

$$
\begin{aligned}
& X_{k+1}=f\left(x_{k}, u_{k}, w_{k}\right) \\
& z_{k}=h\left(x_{k}, v_{k}\right)
\end{aligned}
$$

$$
f\left(x_{k}, u_{k}, w_{k}\right)=\left(\begin{array}{c}
f_{1}\left(x_{k}, u_{k}, w_{k}\right) \\
f_{2}\left(x_{k}, u_{k}, w_{k}\right) \\
\cdot \\
\cdot \\
f_{n}\left(x_{k}, u_{k}, w_{k}\right)
\end{array}\right)
$$

The falling body state vector be $\mathrm{X}_{\mathrm{S}}(\mathrm{k})$ where

$$
\begin{aligned}
& \mathrm{Xs}(\mathrm{k})=\left[\begin{array}{ll}
\mathrm{x} 1(\mathrm{k}) & \mathrm{x} 2(\mathrm{k}) \ldots . . \mathrm{xn}(\mathrm{k})
\end{array}\right]^{\mathrm{T}} \\
& \mathrm{Xs}(\mathrm{k})=\left[\begin{array}{lll}
\mathrm{h}(\mathrm{k}) & \mathrm{v}(\mathrm{k}) & \mathrm{k}
\end{array}\right]^{\mathrm{T}} \\
& \dot{h}(\mathrm{k})=v+w 1
\end{aligned}
$$

$$
\dot{v}(k)=\rho_{0} e^{(-h / k)} *\left(v^{2} / 2 k\right)-g+w 2
$$

$$
\begin{aligned}
& \dot{\boldsymbol{K}}=W_{3}(\boldsymbol{k}) \\
& \mathrm{Z}=\mathrm{h}+\mathrm{V}
\end{aligned}
$$

$$
\begin{array}{ll}
\rho_{0} & - \text { Air density at sea level } \\
\mathrm{g} & - \text { Acceleration due to gravity } \\
\mathrm{W}(\mathrm{k}) & - \text { Process noise } \\
\mathrm{V} & - \text { Measurement noise }
\end{array}
$$

Let $\mathrm{R}(\mathrm{k})$ is the horizontal distance given by

$$
R(k)=\sqrt{M^{2}+h((t)-a)^{2}}+v_{k}
$$

\section{RESUlts}

\section{A. Results}

Fig .4

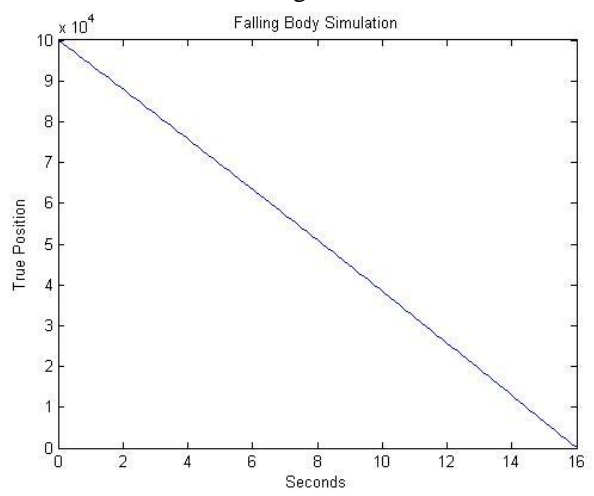

Fig .5

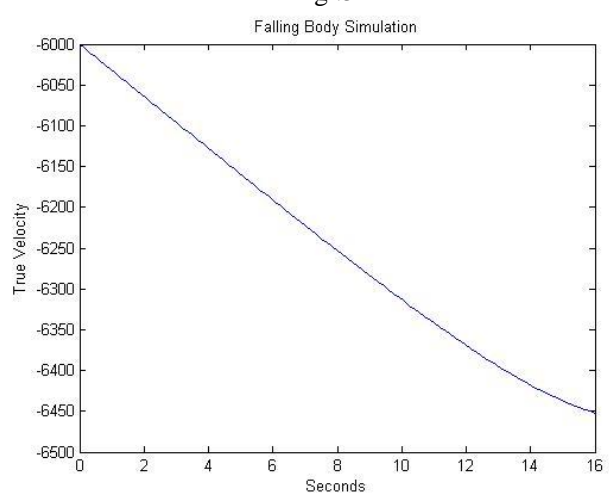

Fig .6 


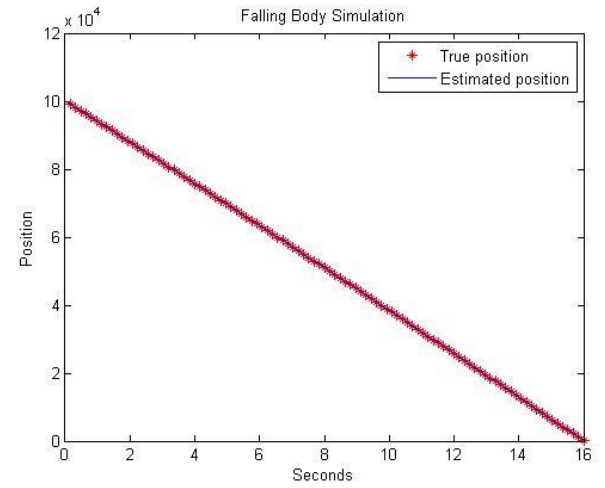

Fig .7

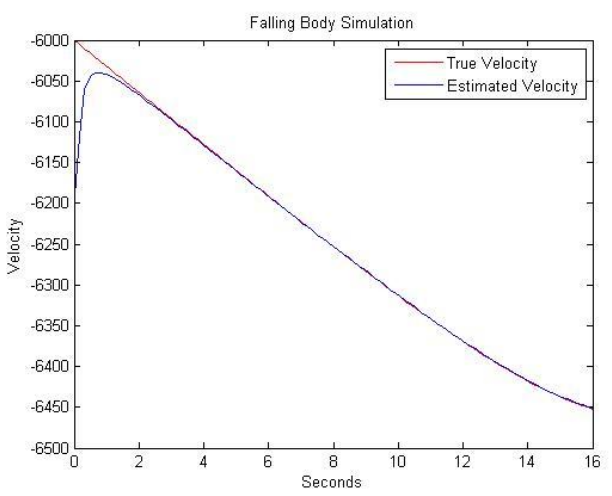

Fig 8

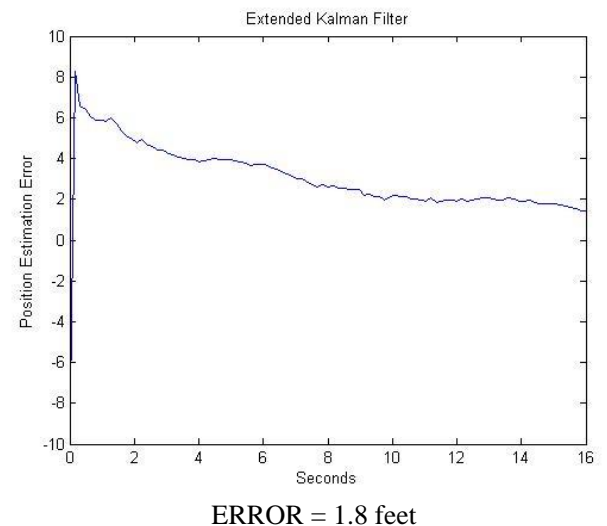

Fig .9

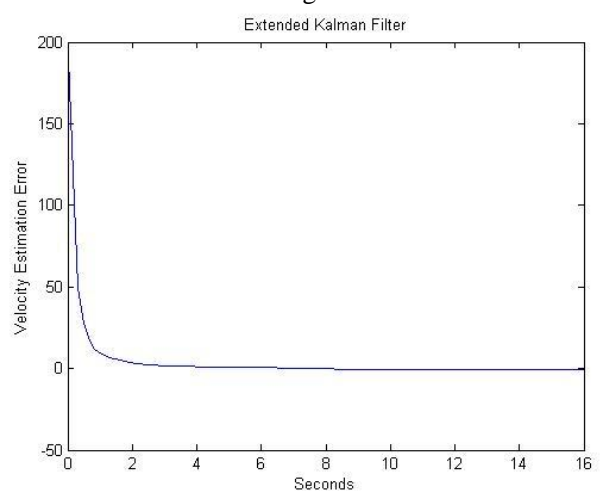

$\mathrm{ERROR}=0 \mathrm{feet} / \mathrm{sec}$
Fig .10

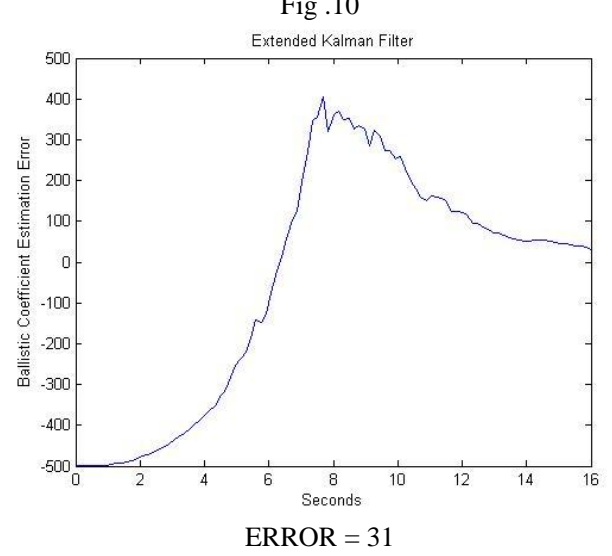

The figures $4, \& 5$ plots the true values of the two parameters Altitude, and velocity respectively and the figures $6 \& 7$ plots the comparision between true and estimated parameters Altitude, and velocity respectively. the figures $8,9, \& 10$ show plots of estimation errors.

\section{CONCLUSIONS}

This paper deals with the performance of Extended Kalman filter which is an extension to the basic Kalman filter. Even though the Kalman filter is simple to implement, it is not able to provide accurate results because it mainly deals with pure linear models. So in order to improve the performance, Extended Kalman filter is implemented for free fall body towards Earth. This model is applied to the non-linear processes. This model deals with linearization of the non-linear process, same as that of the linear approximation. The linearization is upto first order approximation. The linearization of non-linear model can be achieved using Jacobian matrix, which is related to the number of updations. As a result, there will be much more accurate results when compared to that of the Kalman filter. This is applied to a realistic example like tracking a freely falling body. EKF is implemented for a specific application, tracking a freely falling body through the atmosphere for 100 Monte-Carlo simulations and the result shows that EKF provides better probability of state estimation compared to Kalman filter.

\section{FUTURE SCOPE}

The Extended Kalman filter linearizes the nonlinear model through a single point altogether. In addition, if the initial estimate of the state is wrong, or if the process is modeled incorrectly, the filter may quickly diverge, owing to its linearization. Another problem with the extended Kalman filter is that the estimated covariance matrix tends to underestimate the true covariance matrix and therefore risks becoming inconsistent in the statistical sense without the addition of "stabilizing noise". Linearization of nonlinear system with Unscented Kalman filters and particle filters may provide better probability of state estimation.

\section{ACKNOWLEDGMENT}

The authors thank Sri. G.T.Rao, Associate Professor, Department of E.C.E, G.V.P.College of Engineering, for his valuable suggestions in completing this paper. The authors also thank Dr.N.Balasubrhamanyam, Head of the Department 
E.C.E, G.V.P.College of Engineering for the support given in presenting this paper.

\section{REFERENCES}

[1] Simon Julier and Jeffrey Uhlmann. A new extension of the kalman filter to nonlinear systems. Int. Symp. Aerospace/Defense Sensing, Simul. And Controls, Orlando, FL, 1997.

[2] N.J. Gordon, D.J. Salmond, and A.F.M. Smith. A novel approach to nonlinear/non-Gaussian Bayesian state estimation. In IEE Proceedings on Radar and Signal Processing, volume 140, pages 107\{113, 1993.

[3] Leela Kumari B,Padma raju.K,Application of particle filter for a free fall body towards earth,In IJAEA Proceedings ,Volume II,pages $195\{199,2011\}$

[4] Fredrik Orderud Sem Sælands vei 7-9, NO-7491 Trondheim Comparison of Kalman Filter Estimation Approaches for State Space Models with Nonlinear Measurements.

[5] Simon Julier and Jeffrey Uhlmann. A new extension of the kalman filter to nonlinear systems. Int. Symp. Aerospace/Defense Sensing, Simul. And Controls, Orlando, FL, 1997

[6] Cox, H., "On the Estimation of State Variables and Parameters for Noisy Dynamic Systems," IEEE Transactions on Automatic Controls, Vol. AC-9, 1964, pp. 5-12.

[7] M. Athans, R. P. Wishner and A. Bertolini. Suboptimal State Estimation for Continuous-Time Nonlinear Systems from Discrete Noisy Measurements. IEEE Transactions on Automatic Control, TAC13(6):504\{518, October 1968.

[8] F. E. Daum. New Exact Nonlinear Filters. In J. C. Spall, editor, Bayesian Analysis of Time Series and Dynamic Models, chapter 8, pages 199\{226. Marcel Drekker, Inc., 1988

[9] Simon Julier and Jeffrey Uhlmann. A new extension of the kalman filter to nonlinear systems. Int. Symp. Aerospace/Defense Sensing, Simul. And Controls, Orlando, FL, 1997

[10] Extended Kalman Filter_Shoudong ARC Centre of Excellence for Autonomous Systems (CAS) Faculty of Engineering and Information Technology,University of Technology Sydney April 23,2010.

[11] Chi-Tsong Chen. Linear System Theory and Design, third edition. Oxford University Press, 1999.

[12] S. Arulampalam, S. Maskell, N. Gordon, and T. Clapp. A tutorial on particle filters for on-line non-linear/non-Gaussian Bayesian tracking. IEEE Transactions on Signal Processing, 50(2):174-188, February 2002.

[13] N. Bergman, "Recursive Bayesian estimation: Navigation and tracking applications," Ph.D. dissertation, Linköping Univ., Linköping, Sweden,1999..

[14] A. Doucet, "On sequential Monte Carlo methods for Bayesian filtering,” Dept. Eng., Univ. Cambridge, UK, Tech. Rep., 1998.

[15] Leela Kumari B,Padma raju.K, Analysis of state space estimation models for linear and nonlinear systems, In National conference DEVICE-2011 Proceedings ,pages $80\{85,2011\}$

\section{AUTHORS PROFILE}

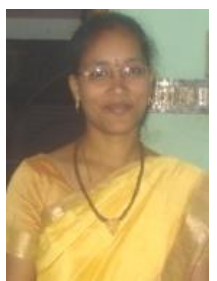

B.Leela Kumari received the B.Tech degree in Electronics and Communication Engineering from J.N.T.University, and M.Tech degree in Radar and
Microwave Engineering from Andhra University College of Engineering. She has 8 years of teaching experience and is Associate Professor of Electronics and Communication Engineering, G.V.P.College of Engineering, Visakhapatnam. She has published 7 technical papers in National/International Journals/Conference proceedings.Her research interests include Signalprocessing, State Estimation, tracking

and particle filters. She registered for Ph.D. from J.N.T.U. Kakinada.

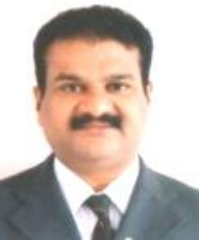

K.Padma Raju received B.Tech from Nagarjuna University, M. Tech from NIT Warangal, Ph. D from Andhra University, India and Post Doctoral Fellowship at Hoseo University, South Korea. He has worked as Digital Signal Processing Software Engineer in Signion Systems Pvt. Ltd., Hyderabad, India, before joining Jawaharlal Nehru Technological University Kakinada, India.

He has 17 years of teaching experience and is Professor of Electronics andCommunication Engineering, Jawaharlal Nehru Technological University Kakinada, India. Presently working as Director, Industry Institute Interaction, Placements \& Training, Jawaharlal Nehru Technological University Kakinada, India.He worked as Research Professor at Hoseo University, South Korea during 2006-2007. He has published 30 technical papers in National/International Journals/Conference proceedings and guiding 06 research students in the area of Antennas, EMI/EMC and Signal Processing.His fields of interest are Signal Processing, Microwave and Radar Communications and EMI/EMC.

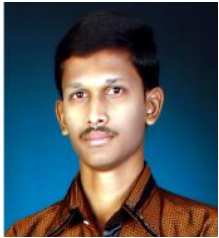

V.Y.V.Chandan received the B.Tech degree in Electronics and Communication Engineering from J.N.T.University. His research interests include Signal processing, State Estimation.

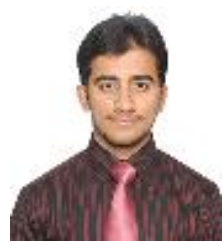

R.Sai Krishna received the B.Tech degree in Electronics and Communication Engineering from J.N.T.University, His research interests include Signal processing, State Estimation.

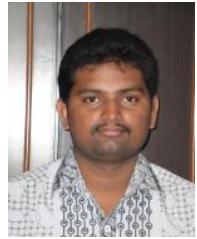

V.M.J.Rao received the B.Tech degree in Electronics and Communication Engineering from J.N.T.University, His research interests include Signalprocessing, State Estimation. 